\title{
Relationship Between Angiographically Documented Coronary Artery Disease and Low Bone Mass in Men
}

\author{
Enver Erbilen, MD; Selma Yazici, MD*; Hakan Özhan, MD; \\ Serkan Bulur, MD; Serkan Ordu, MD; Mehmet Yazici, MD
}

\begin{abstract}
Background The present study aimed to investigate the association between low bone mass (LBM) and coronary artery disease (CAD) in male patients.

Method and Results The data for 47 men who were screened for osteopenia and osteoporosis with dual energy X-ray absorptiometry and then underwent coronary angiography between February 2005 and May 2006 were retrospectively analyzed. Bone mineral density of the femur neck was stratified as normal (T score $>-1.0 \mathrm{SD}$ ) or low (T score $<-1.0 \mathrm{SD}$ ) and CAD was defined as $\geq 50 \%$ occlusion in at least 1 major coronary artery. Thirty-two patients were found to have angiographically significant CAD. Patients in the LBM group had a significantly higher incidence of CAD. Low bone mass was significantly and positively correlated with the Gensini scores of the patients $(\mathrm{r}=0.6037, \mathrm{p}<0.0001)$ and was found to be an independent predictor of $\mathrm{CAD}$ in multivariate logistic regression analysis (odds ratio: 5.4 [95\% confidence interval 1.66 and 17.49]; $\mathrm{p}=0.0049$ ). Repeated statistical analyses with the acceptance of CAD as coronary artery stenosis $>75 \%$ confirmed the same results.
\end{abstract}

Conclusion Low bone mass was significantly associated with angiographically documented CAD in males. (Circ J 2007; 71: 1095-1098)

Key Words: Bone mass; Coronary artery disease; Males

$\mathbf{L}$ ow bone mass (LBM) has been found to be independently associated with cardiovascular events.-4 There was an increased incidence of cardiovascular events over a 4-year follow-up in female patients who had LBM at baseline ${ }^{2}$ and Von der Recke et al found a 2-fold increase in cardiovascular mortality in postmenopausal women who were categorized within the lowest quartile of bone mineral density? A correlation between LBM and the incidence of coronary artery disease (CAD) was observed in women in the Framingham study 5 . All of these studies were conducted in predominantly female patients and the association between LBM and CAD in male patients has not been studied, so in the present study we investigated the association of bone mineral density (BMD) and CAD diagnosed with coronary angiography in male patients who had been screened for osteopenia and osteoporosis.

\section{Methods}

\section{Patients}

The current study, which was carried out in the departments of Cardiology and Physical Medicine of Düzce University Faculty of Medicine, was based on a retrospective analysis of 3,104 patients screened for osteopenia or osteoporosis by dual energy X-ray absorptiometry (DXA)

(Received November 13, 2006; revised manuscript received March 7, 2007; accepted March 28, 2007)

Departments of Cardiology, *Physical Medicine and Rehabilitation, Faculty of Medicine, Düzce University, Düzce, Turkey

Mailing address: Hakan Özhan, MD, Department of Cardiology,

Faculty of Medicine, Düzce University, Düzce Tïp Fakültesi Kardiyoloji Klinigi, Konuralp Düzce 81620, Turkey. E-mail: ozhanhakan@yahoo.com between February 2005 and May 2006. Sixty-seven patients without any exclusion criteria (20 females, 47 males) were found to have had coronary angiography and DXA scans in the same period and only the 47 males, with a mean age of $66 \pm 9$ years, were included in the study. Exclusion criteria were acute coronary syndrome during the past 4 weeks, acute state of a chronic infectious or inflammatory disease, anticoagulant therapy, severe liver or renal disease, neoplasm, hematological disorders, and physical inactivity. The Institutional Review Board of the Düzce University Faculty of Medicine approved the study, a waiver of consent was granted and patient identity was protected.

Prior medical histories, including comorbidities that might affect bone density, were recorded. Diuretic and steroid use, both known to affect BMD, were also recorded. Biochemical data were collected from the patients' physical medicine files, and included fasting glucose and levels of cholesterol, creatinine, albumin, calcium and magnesium.

\section{Angiography}

Coronary angiography was performed by the Judkins method. Two skilled cardiologists who were unaware of the clinical and laboratory data scored the coronary angiograms. Patients who had $\geq 50 \%$ occlusion in at least 1 major coronary artery were classified as having CAD. Coronary artery trees of every patient were counted and scored using the Gensini method6

\section{BMD Scans}

$\operatorname{BMD}\left(\mathrm{g} / \mathrm{cm}^{2}\right)$ was measured for the lumbar spine (L2 to L4), proximal femur, and 1/3 distal radius using a GELunar DPX-NT PRO (Lunar Corp, Adison, WI, USA) by DXA scanning. BMD was determined according to stan- 
Table 1 Comparison of the Characteristics of Patients With Significant or Insignificant CAD

\begin{tabular}{lccc}
\hline \hline & $\begin{array}{c}C A D \\
(n=32)\end{array}$ & $\begin{array}{c}\text { Non-CAD } \\
(n=15)\end{array}$ & p value \\
\hline Age (years) & $65 \pm 10$ & $63 \pm 7$ & 0.595 \\
BMI $\left(\mathrm{kg} / \mathrm{m}^{2}\right)$ & $27 \pm 3$ & $30 \pm 3$ & 0.049 \\
Hypertension $(\mathrm{n})(\%)$ & $8(25 \%)$ & $5(33 \%)$ & 0.552 \\
Smoker $(\mathrm{n})(\%)$ & $11(34 \%)$ & $5(33 \%)$ & 0.944 \\
DM $(\mathrm{n})(\%)$ & $12(40 \%)$ & $8(53 \%)$ & 0.396 \\
Hyperlipidemia $(\mathrm{n})(\%)$ & $8(26 \%)$ & $6(40 \%)$ & 0.327 \\
Statin use $(\mathrm{n})(\%)$ & $8(25 \%)$ & $5(33 \%)$ & 0.552 \\
ASA use $(\mathrm{n})(\%)$ & $31(97 \%)$ & $13(86 \%)$ & 0.182 \\
TC $(\mathrm{mg} / \mathrm{dl})$ & $168 \pm 50$ & $172 \pm 37$ & 0.798 \\
HDL-C $(\mathrm{mg} / \mathrm{dl})$ & $51 \pm 21$ & $46 \pm 18$ & 0.551 \\
LDL-C $(\mathrm{mg} / \mathrm{dl})$ & $95 \pm 35$ & $96 \pm 28$ & 0.928 \\
Fasting glucose $(\mathrm{mg} / \mathrm{dl})$ & $117 \pm 40$ & $122 \pm 43$ & 0.705 \\
Creatinine $(\mathrm{mg} / \mathrm{dl})$ & $1.0 \pm 0.26$ & $1.1 \pm 0.14$ & 0.542 \\
Calcium $(\mathrm{mg} / \mathrm{dl})$ & $9.1 \pm 0.5$ & $9.2 \pm 0.4$ & 0.764 \\
Phosphorus $(\mathrm{mg} / \mathrm{dl})$ & $3.2 \pm 0.6$ & $3.4 \pm 0.5$ & 0.859 \\
Albumin $(\mathrm{mg} / \mathrm{dl})$ & $4.1 \pm 0.3$ & $4.3 \pm 0.2$ & 0.09 \\
\hline
\end{tabular}

$C A D$, coronary artery disease; BMI, body mass index; DM, diabetes mellitus; ASA, aspirin; TC, total cholesterol; $H D L-C$, high-density lipoproteincholesterol; LDL-C, low-density lipoprotein-cholesterol.

Table 2 Comparison of the T Scores of the 2 Patient Groups

\begin{tabular}{lccc}
\hline \hline & $\begin{array}{c}C A D \\
(n=32)\end{array}$ & $\begin{array}{c}\text { Non-CAD } \\
(n=15)\end{array}$ & p value \\
\hline $\begin{array}{lcc}\text { Low bone mass } \\
\quad(\text { T score }<-1)(n)\end{array}$ & $18(56 \%)$ & $1(7 \%)$ & 0.001 \\
Femoral & $-1.1 \pm 1$ & $0.02 \pm 1.0$ & 0.001 \\
Lumbar & $-0.8 \pm 1.3$ & $0.7 \pm 1.8$ & 0.002 \\
Radius & $-0.1 \pm 1.3$ & $0.8 \pm 2.1$ & 0.123 \\
Diseased vessels & $1 \pm 0.8$ & $0 \pm 0$ & $<0.001$ \\
\hline
\end{tabular}

Abbreviation see in Table 1.

dard Lunar protocols. BMD results for the femur neck were classified into 3 groups according to World Health Organization criteria: ${ }^{7}$ normal (T score $>-1.0 \mathrm{SD}$ ), osteopenia (T score -1.0 to $-2.5 \mathrm{SD}$ ) and osteoporosis (T score $<-2.5 \mathrm{SD}$ ). Patients with osteopenia or osteoporosis ( $\mathrm{T}$ score $<-1.0$ SD) were grouped as having LBM.

Statistical Analysis

Values were presented as mean \pm standard deviation.
Table 3 Independent Predictors of CAD (Logistic Regression Analyses)

\begin{tabular}{lllll}
\hline \hline \multirow{2}{*}{ OR } & \multicolumn{2}{c}{$95 \%$ CI } & \multirow{2}{*}{ p value } \\
\cline { 3 - 4 } & & Lower & Upper & \\
\hline \multirow{2}{*}{ Age } & 1.01 & 0.93 & 1.10 & 0.7 \\
Smoking status & 1.1 & 0.2 & 5.87 & 0.911 \\
Hypertension & 1.76 & 0.24 & 12.68 & 0.572 \\
DM & 0.14 & 0.017 & 1.2 & 0.074 \\
Hyperlipidemia & 0.87 & 0.15 & 5.05 & 0.882 \\
Low bone mass & 22.55 & 2.07 & 244.69 & 0.01 \\
\hline
\end{tabular}

OR, odds ratio; CI, confidence interval. Other abbreviations see in Tables 1,2.

Table 4 Comparison of T scores of Patients When Grouped According to Coronary Stenosis $>\mathbf{7 5 \%}$

\begin{tabular}{lccc}
\hline \hline & $\begin{array}{c}C A D \\
(n=24)\end{array}$ & $\begin{array}{c}\text { Non-CAD } \\
(n=23)\end{array}$ & p value \\
\hline $\begin{array}{l}\text { Low bone mass } \\
\quad(\text { T score }<-1)(n)\end{array}$ & $15(63 \%)$ & $4(17 \%)$ & 0.002 \\
Femoral & $-1.2 \pm 1$ & $-0.2 \pm 1.0$ & 0.003 \\
Lumbar & $-1.1 \pm 1.2$ & $0.6 \pm 2$ & 0.001 \\
Radius & $-0.3 \pm 1$ & $0.7 \pm 2$ & 0.078 \\
Diseased vessel number & $1.3 \pm 0.7$ & $0 \pm 0$ & $<0.001$ \\
\hline
\end{tabular}

Abbreviation see in Table 1.

Normal distribution was controlled with Mann-Whitney Utest. Student's t-test was used to compare continuous variables. Categorical data were compared with chi-square test. Spearmen's correlation test was used to assess correlations between LBM and CAD. Linear regression analysis was performed to examine the association between Gensini and T scores. Multivariate logistic regression analysis was used to assess the independent association of BMD with CAD. Statistical analyses were carried out using SPSS 10.0 for Windows and a p-value $<0.05$ was considered significant

\section{Results}

Of the 47 patients 32 had angiographically significant CAD and 15 had insignificant stenosis and were classified as the non-CAD group. Demographic, clinical and laboratory variables of the patients with and without CAD are shown in Table 1. Demographic variables were not signifi-

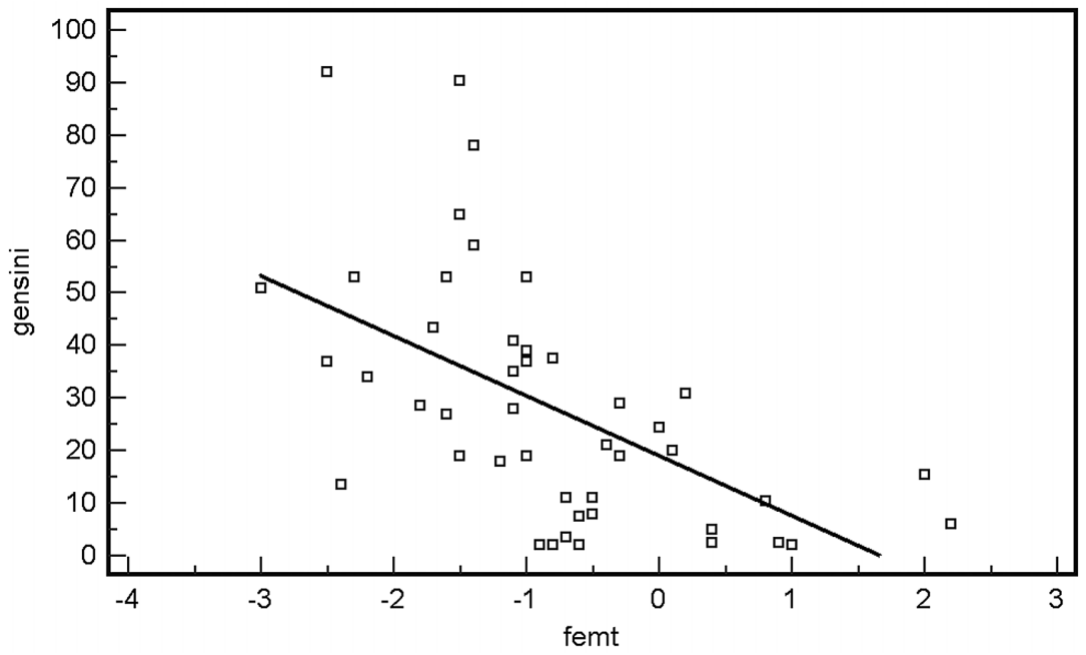

Fig 1. Regression line on the scatter graph of the coronary Gensini scores with femur neck $\mathrm{T}$-scores in male patients (gensini [Y axis]: coronary Gensini score, femt [X axis]: T scores of femur neck $r=-0.57, \mathrm{p}<0.0001$ ). 
cantly different between groups, excepting mean body mass index, which had only borderline significance. Comparisons of the BMD indices between groups are shown in Table 2. The number of diseased vessels was higher and the $\mathrm{T}$ scores for the lumbar spine and femur neck were significantly lower in patients with CAD. LBM was also significantly correlated with CAD $(r=0.38, p=0.002)$. Femur neck T-scores showed a significant inverse linear relation with Gensini score $(r=-0.57, \mathrm{p}<0.0001)($ Fig 1$)$.

Stepwise multivariate logistic regression analysis was used to identify independent determinants of CAD (Table 3). The covariates considered were LBM, age, gender, smoking status, hypertension, diabetes mellitus and hyperlipidemia. LBM was found to be an independent predictor of CAD (odds ratio: 22.55 [95\% confidence interval: 2.072 and 244.69]; $\mathrm{p}=0.01$ ).

All the statistical analyses were repeated with acceptance of CAD as coronary artery stenosis $>75 \%$. Comparisons of the BMD indices were not different (Table4) and LBM was still an independent predictor of CAD (odds ratio: 8.27 [95\% confidence interval: 1.90 and 36.03]; $\mathrm{p}=0.005$ ).

\section{Discussion}

This retrospective cross-sectional study is the first to demonstrate an association between low BMD and angiographically documented CAD in a population consisting only of male patients. We found LBM to be independently and significantly related with $\mathrm{CAD}$ in logistic regression analysis and specifically confirmed this in the inverse relationship of femoral T scores with coronary Gensini scores.

The present findings support those of previous studies, which reported an association between low BMD and cardiovascular events ${ }^{1-4}$ and $\mathrm{CAD}^{5,8,9}$ Uyama et al reported a significant correlation between the severity of carotid atherosclerosis detected with duplex ultrasound and BMD of the proximal femur in postmenopausal women? Marcovitz et al found similar results in a population consisting predominantly of women. They retrospectively analyzed 209 ambulatory adult patients ( $89 \%$ female) who were referred for DXA scans by their primary care physician and underwent coronary angiography within the same 1-year period. Low BMD appeared to independently predict significant CAD in women, with a higher odds ratio than traditional risk factors? Our findings; besides confirming the findings in women, further conclude a similar association in men, which is the first report of LBD as a predictor for CAD in men.

The pathophysiologic mechanism underlying low BMD in association with $\mathrm{CAD}$ is still unclear. However, emerging clinical and molecular evidence suggests that inflammation exerts a significant influence on bone turnover, inducing osteoporosis. Numerous proinflammatory cytokines have been implicated in the regulation of osteoblasts and osteoclasts, and a shift towards an activated immune profile has been hypothesized as an important risk factor ${ }^{10}$ Under the influence of lifelong exposure to chronic antigenic load and oxidative stress, the physiological counter-regulatory process that inhibits bone resorption following T-cell activation is likely impaired and would contribute, together with systemic low-grade inflammation, to the increasing incidence of osteoporosis $!^{10}$ In fact, atherosclerosis and osteoporosis seem to share a common pathogenesis, such as bone matrix proteins, bone morphogenetic protein and inflammatory proteins. Clinical studies will further enlighten the exact relationship, but at present such studies are very few!1-14 The degree of coronary calcium as assessed by electron beam computed tomography was found to be inversely correlated with BMD, thus substantiating the relationship between bone demineralization and the total coronary atherosclerotic burden!2 Sinnot et al found that coronary calcification was inversely associated with BMD in postmenopausal women, using univariate analysis. Using multivariate logistic regression analysis they found age as the only significant predictor of positive coronary calcification and low BMD $!^{13}$ In a rat model, Smith et al demonstrated that systemic bone loss may induce coronary vessel disease ${ }^{14}$ What is more surprising is that after Trichrome staining of the coronary arteries, they found significant changes consistent with vascular disease. Another common factor explaining the apparent association between atherosclerosis and bone loss may be the presence of oxidized lipids, which promote atherogenesis ${ }^{15}$ and inhibit differentiation and mineralization of bone cells! 16

Supporting the role played by estrogen deficiency in women, the relationship between aortic calcification and bone loss seems to be strongest during the early phase of menopause, characterized by a rapid decrease in circulating estrogen ${ }^{17}$ Estrogen $\alpha$ - and $\beta$-receptor subtypes are also expressed in men in the osteoblasts, as well as in chondrocytes ${ }^{18}$ Furthermore, BMD and the prevalence of vertebral fractures are positively correlated with serum estrogen concentrations in men. ${ }^{9}$ These results acknowledge the possible link between a reduced amount of estrogen secretion and the underlying mechanism shared by impaired bone metabolism and atherosclerosis in men. Unfortunately, as a retrospective study with known constraints, we could not investigate the serum estrogen levels, which would possibly have enhanced our findings.

Another relationship between LBM and CAD is the existence of a similar risk profile. Several well-known atherosclerotic risk factors are also associated with low BMD; for instance, exercise, a well known positive life style change for atherosclerosis, has been found to increase the lumbar spine BMD?20 Another interesting finding linking the pathophysiology of LBM and atherosclerosis was published by Ortlepp et $\mathrm{al}^{21}$ who found that a common genetic polymorphism of the vitamin $\mathrm{D}$ receptor, which has been found to be associated with BMD, was also associated with diabetes mellitus types I and II and CAD. There are also reports of testosterone supplementation in older males that has improved exercise-induced coronary ischemia and lumbar BMD. Further investigations will enlighten the absolute paths that link LBM with atherosclerosis.

\section{Study Limitations}

Potential limitation of this retrospective cross-sectional study are the relatively small number of patients, the restricted records of confounding factors (ie, socioeconomic status, dietary habits) in this cohort and the possible influence of a reverse causality bias (ie, CAD causing a reduction in BMD through some mechanism rather than necessarily LBM causing CAD) because of the cross-sectional design. Therefore, large-scale prospective studies are needed to understand whether these results apply clinically and to determine whether treatment of low BMD will modify the severity and progression of $\mathrm{CAD}$ in the general population.

Finally, we conclude that LBM is significantly associated with angiographically documented CAD, and the effect is independent of conventional risk factors. 


\section{References}

1. Browner WS, Pressman AR, Nevitt MC, Cauley JA, Cummings SR. Study of Osteoporotic Fractures Research Group: Association between low bone density and stroke in elderly women: The study of osteoporotic fractures. Stroke 1993; 24: 940-946.

2. Tanko LB, Christiansen C, Cox DA, Geiger MJ, McNabb MA, Cummings SR. Relationship between osteoporosis and cardiovascular disease in postmenopausal women. J Bone Miner Res 2005; 20: $1912-1920$.

3. Von der Recke P, Hansen MA, Hassager C. The association between low bone mass at the menopause and cardiovascular mortality. Am J Med 1999; 106: $273-278$.

4. Jørgensen L, Engstad T, Jacobsen BK. Bone mineral density in acute stroke patients: Low bone mineral density may predict first stroke in women. Stroke 2001; 32: 47-51.

5. Samelson EJ, Kiel DP, Broe KE, Zhang Y, Cupples LA, Hannan MT, et al. Metacarpal cortical area and risk of coronary heart disease: The Framingham Study. Am J Epidemiol 2004; 159: 589-595.

6. Gensini GG. A more meaningful scoring system for determining the severity of coronary heart disease. Am J Cardiol 1983; 51: 606-607.

7. Cummings SR, Bates D, Black DM. Clinical use of bone densitometry. JAMA 2002; 288: 1889-1897.

8. Uyama O, Yoshimoto Y, Yamamoto Y, Kawai A. Bone changes and carotid atherosclerosis in postmenopausal women. Stroke 1997; 28: $1730-1732$

9. Marcovitz PA, Tran HH, Franklin BA, O'Neill WW, Yerkey M, Boura J, et al. Usefulness of bone mineral density to predict significant coronary artery disease. Am J Cardiol 2005; 96: 1059-1063.

10. Ginaldi L, Di Benedetto MC, De Martinis M. Osteoporosis, inflammation and ageing. Immun Ageing 2005; 2: 14

11. Hak AE, Pols HA, van Hemert AM, Hofman A, Witteman JC. Progression of aortic calcification is associated with metacarpal bone loss during menopause: A population-based longitudinal study. Arterioscler Thromb Vasc Biol 2000, 20: 1926-1931.

12. Barengolts EI, Berman M, Kukreja SC, Kouznetsova T, Lin C,
Chomka EV. Osteoporosis and coronary atherosclerosis in asymptomatic postmenopausal women. Calcif Tissue Int 1998; 62: 209-213.

13. Sinnott B, Syed I, Sevrukov A, Barengolts E. Coronary calcification and osteoporosis in men and postmenopausal women are independent processes associated with aging. Calcif Tissue Int 2006; 78: 195-202.

14. Smith BJ, Lerner MR, Bu SY, Lucas EA, Hanas JS, Lightfoot SA, et al. Systemic bone loss and induction of coronary vessel disease in a rat model of chronic inflammation. Bone 2006; 38: 378-386.

15. Witztum JL, Steinberg D. Role of oxidized low density lipoprotein in atherogenesis. J Clin Invest 1991; 88: 1785-1792.

16. Parhami F, Morrow AD, Balucan J, Leitinger N, Watson AD, Tintut Y, et al. Lipid oxidation products have opposite effects on calcifying vascular cell and bone cell differentiation: A possible explanation for the paradox of arterial calcification in osteoporotic patients. Arterioscler Thromb Vasc Biol 1997; 17: 680-687.

17. Hak AE, Pols HA, van Hemert AM. Progression of aortic calcification is associated with metacarpal bone loss during menopause: A population-based longitudinal study. Arterioscler Thromb Vasc Biol 2000; 20: 1926-1931.

18. Byers RJ, Hoyland JA, Braidman IP. Osteoporosis in men: A cellular endocrine perspective of an increasingly common clinical problem. $J$ Endocrinol 2001; 168: 553-562.

19. Barrett-Connor E, Mueller JE, von Muhlen DG, Laughlin GA, Schneider DL, Sartoris DJ. Low levels of estradiol are associated with vertebral fractures in older men, but not women: The Rancho Bernardo Study. J Clin Endocrinol Metab 2000; 85: 219-223.

20. Neville CE, Murray LJ, Boreham CA, Gallagher AM, Twisk J, Robson PJ, et al. Relationship between physical activity and bone mineral status in young adults: The Northern Ireland Young Hearts Project. Bone 2002; 30: 792-798.

21. Ortlepp JR, Lauscher J, Hoffmann R, Hanrath P, Joost HG. The vitamin $\mathrm{D}$ receptor gene variant is associated with the prevalence of type 2 diabetes mellitus and coronary artery disease. Diabet Med 2001; 18: 842-845. 